\title{
Generation and Termination of Stacking Faults by Inverted Domain Boundaries in 3C-SiC
}

Massimo Zimbone, Eric Gasparo Barbagiovanni, Corrado Bongiorno, Cristiano Calabretta, Lucia Calcagno, Giuseppe Fisicaro, Antonino La Magna, Francesco La Via 
Figure 1 S.I.

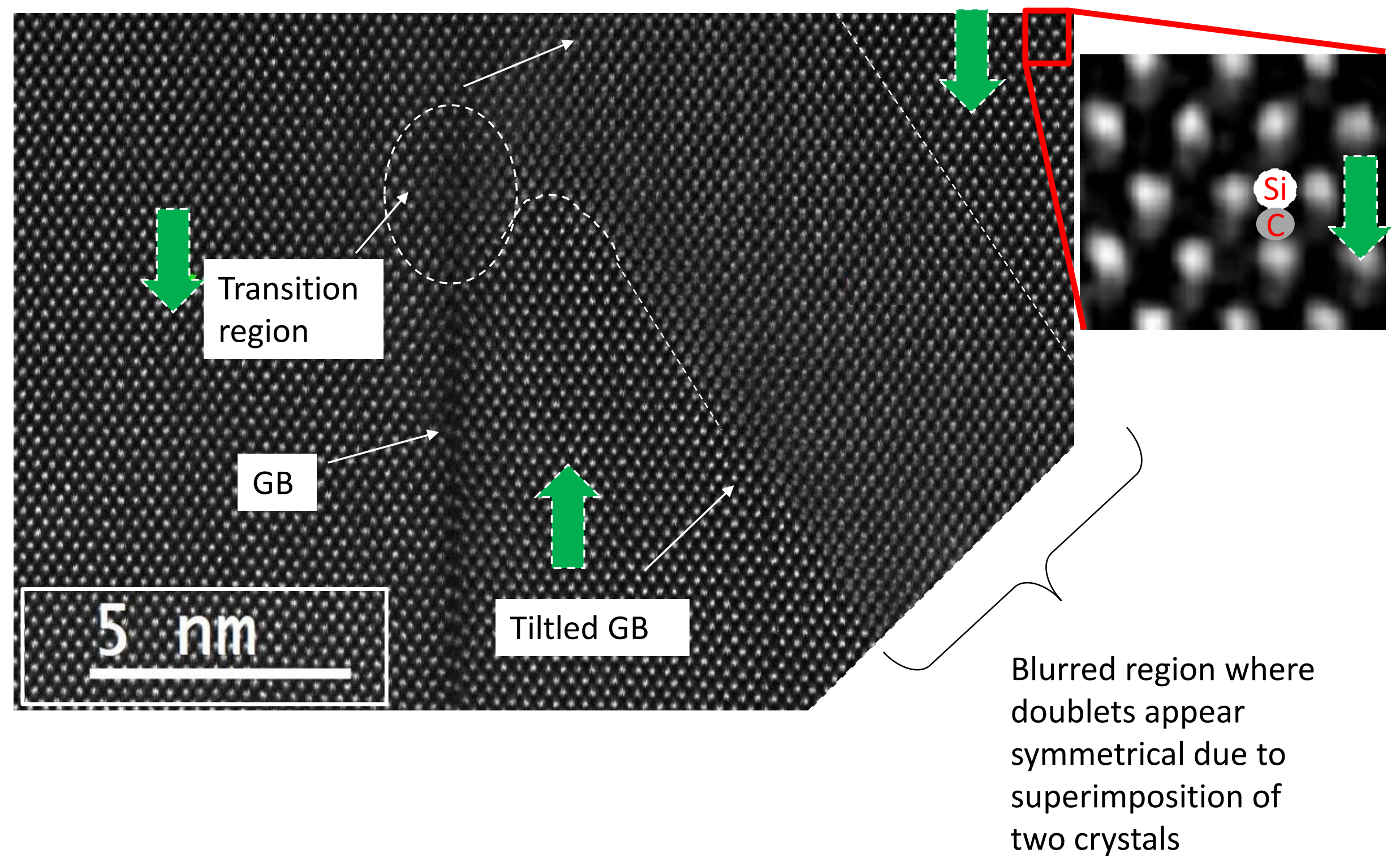


Figure 2 S.I.

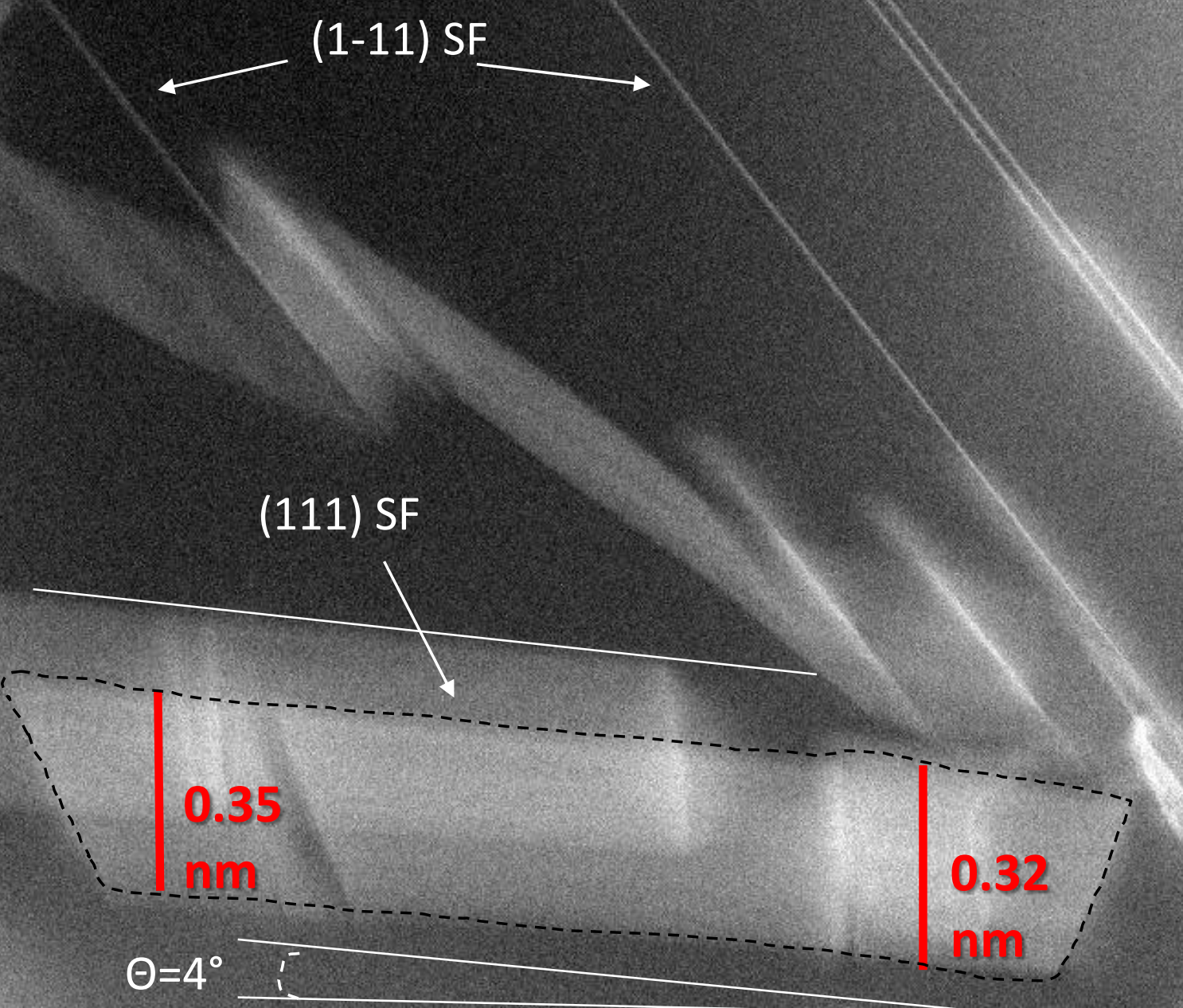

Thickness of the lamella is almost uniform as is depicted from the size of the SF 0.35 and $0.32 \mathrm{~nm}$.

Nevertheless it is slightly mis oriented ( $4^{\circ}$ respect to $<1-10>$ ) meaning that lamella is $4^{\circ}$ off respect to (110) plane. 
Intersection of (1-11) plane with lamellae in (110) plane gives the yellow line.
Intersection of (011)

plane with lamellae (110)

gives the red line.
Figure 3 S.I.

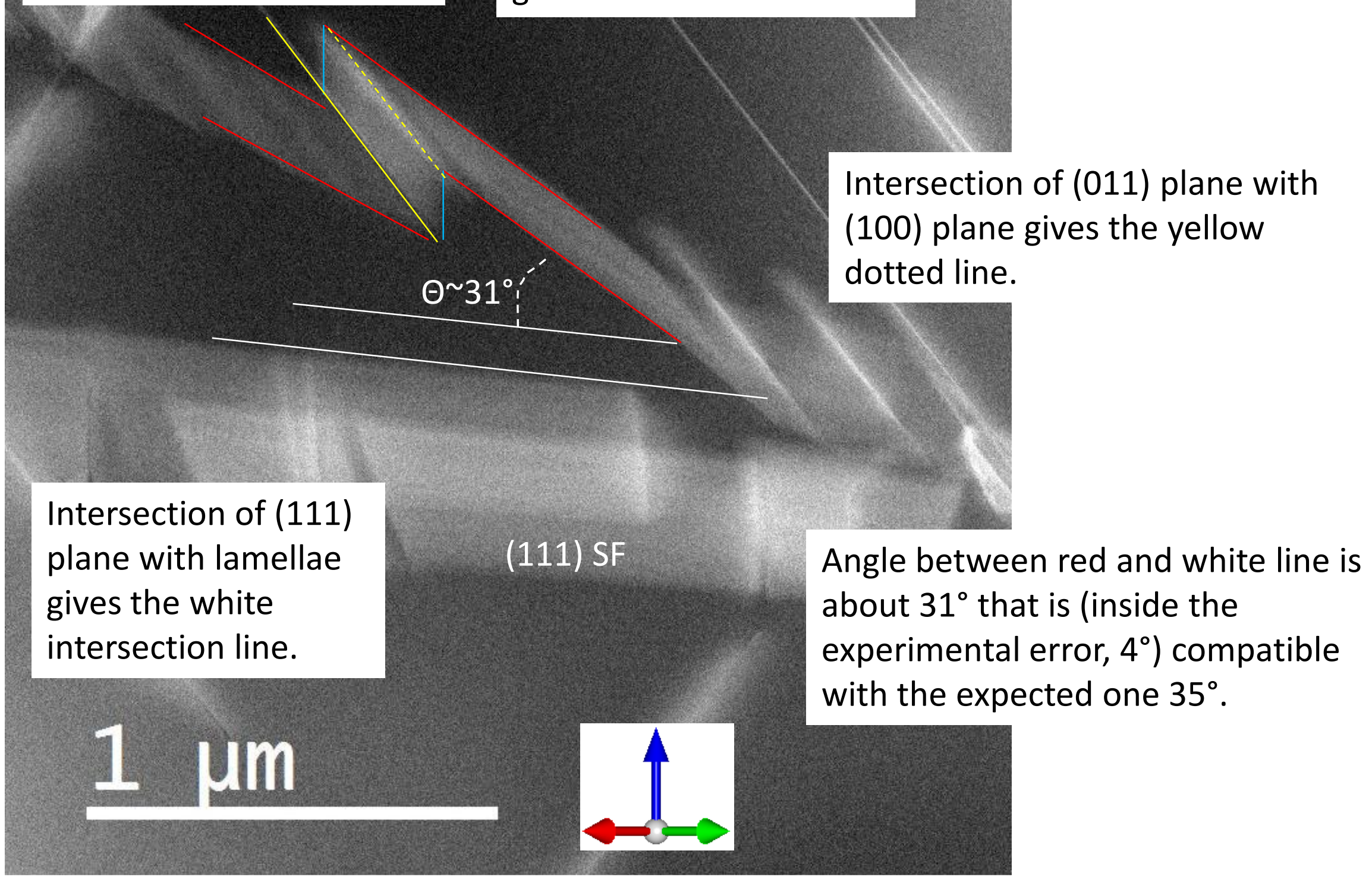


Figure 4 S.I.

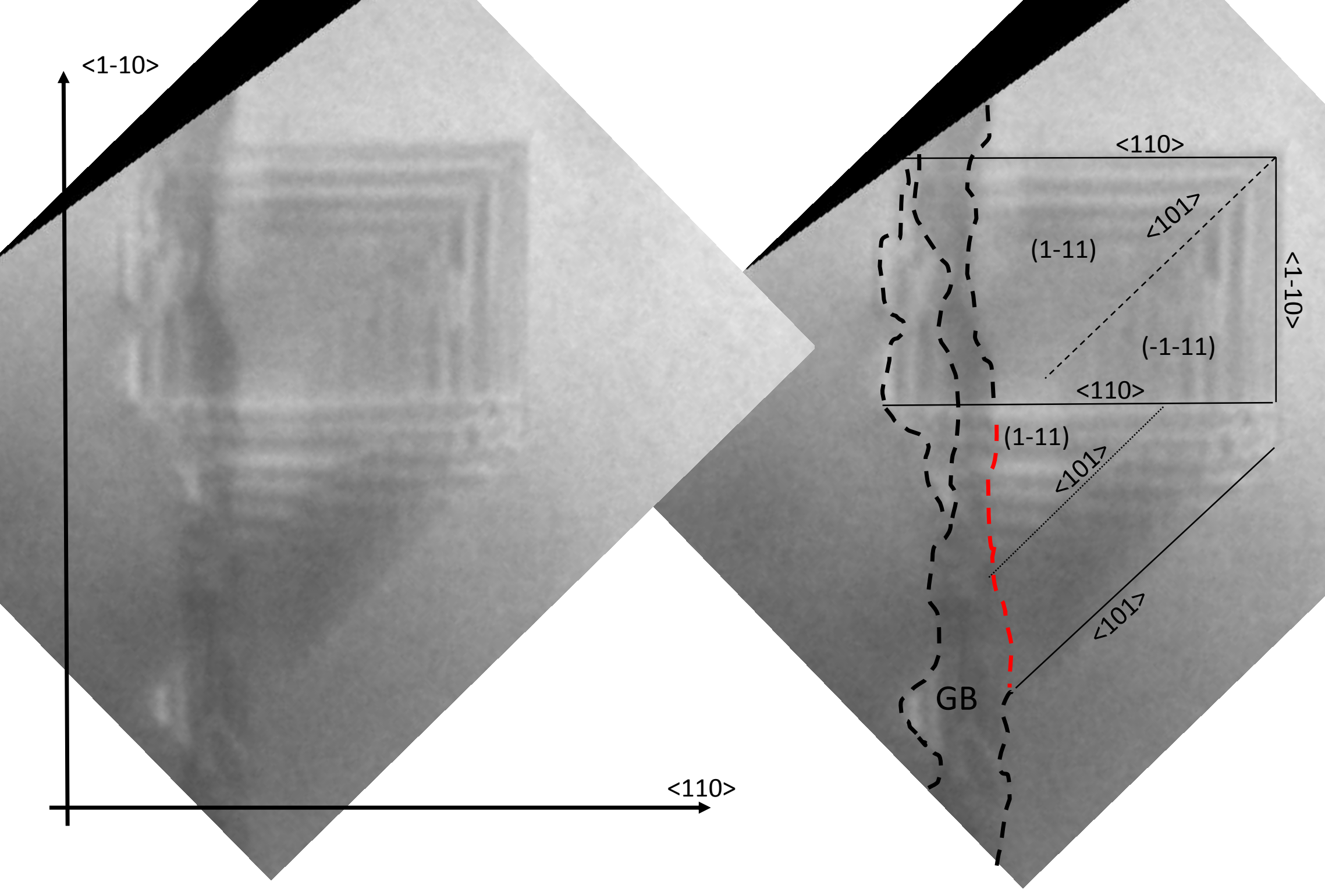


Figure 5 S.I.

FREE SFs ZONE

\section{FULL SFs ZONE}




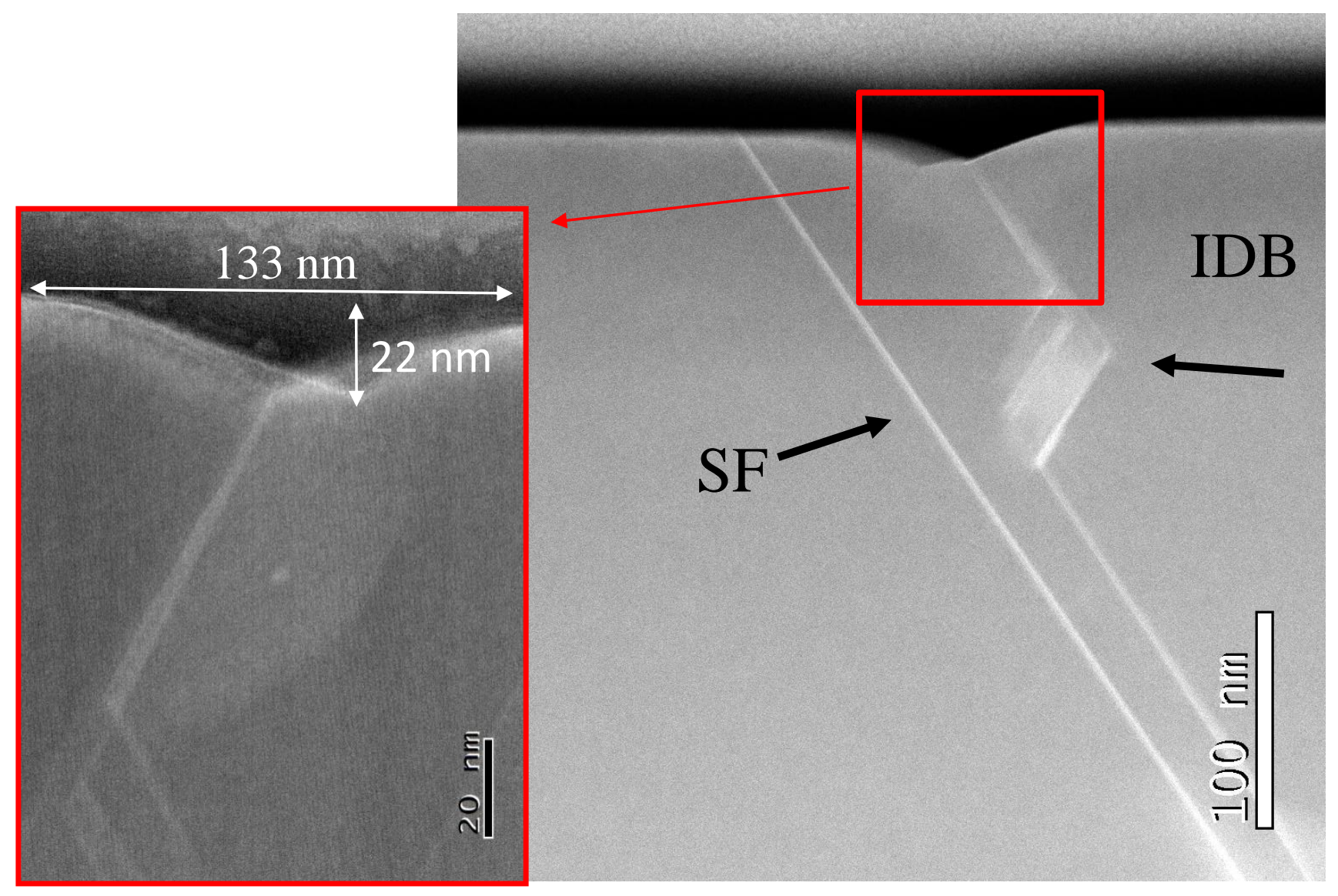

Figure 6 S.I. 